\title{
An Efficient and Environmentally Friendly Procedure for Synthesis of Quinazolinone Derivatives by Use of a Biginelli-Type Reaction
}

\author{
NADIA T. A. DAWOUD* \\ Department of Chemistry, Faculty of Science "Girls”, \\ Al-Azhar University, Nasr City, Cairo, Egypt \\ dawoudnadia@yahoo.com
}

Received 4 August 2012 / Accepted 4 September 2012

\begin{abstract}
A Biginelli one-pot three-component reaction involving cyclohexanone, aromatic/or heterocyclic aldehyde, and urea thiourea or guanidine $\mathrm{HCl}$ was applied in this work to prepare quinazolin-2(1H)one derivatives under solvent free and microwave irradiation assistance as well as conventional method. The major advantages of this protocol are high yields, operational simplicity, short reaction time and environment friendly. The purity of the synthesized compounds was characterized by means of IR, ${ }^{1} \mathrm{H}$ NMR, ${ }^{13} \mathrm{C}$ NMR, mass spectral and elemental analysis.
\end{abstract}

Keywords: Multi-component reactions, Microwave assisted synthesis, Quinazolin-2(1H)-one derivatives, Green chemistry

\section{Introduction}

Multi-component reactions (MCRs) are becoming increasingly prevalent due to their improved efficiency, simple procedure, one-pot character, quantitative yields of the target molecules and the high and ever increasing number of accessible backbones. The centuryold Biginelli reaction as a classical multi component reaction has gained much importance inorganic synthesis, partly because of the diverse types of physiological activity associated with the dihydropyrimidinones produced by this reaction. The classical Biginelli reaction is a simple one-pot cyclondensation of $\beta$ - dicarbonyl compounds with aldehydes, urea, or thiourea in the presence of various catalysts ${ }^{1-16}$. An efficient alternative for synthesis of fused pyrimidinones by a three-component condensation with aromatic aldehyde, cyclopentanone and urea or thiourea was reported ${ }^{17}$. The development of a simple, efficient and environmentally friendly method for the Biginelli-type reactions needed. Here in, the present investigation deals with a novel and efficient procedure for a new green synthetic method of fused pyrimidinone derivatives via three-component cyclocondensation of aromatic or heterocyclic aldehydes, cyclohexanone, with urea, thiourea, or guanidine under neat conditions (Scheme 1) or under solvent free and microwave irradiation (Scheme 2). 


\section{Experimental}

All commercial reagents and solvents were used without purification. Reactions were monitored by TLC performed on silica gel plates (Merck). Melting points (Uncorrected) were measured in open capillary tubes. The IR spectra were recorded on Perkin -Elmer-843 spectrometer using $\mathrm{KBr}$ pellets. ${ }^{1} \mathrm{H}$ NMR and ${ }^{13} \mathrm{C}$ NMR spectra were recorded on BRUKER AVANCE II 400 NMR spectrometer inCDCl 3 ; chemical shifts (d) were expressed in ppm relative to tetramethylsilane used as an internal standard.

\section{Biginelli conventional method}

Synthesis of 8-(substituted-arylidene)-4-(substituted-phenyl)quinazolin-2(1H)one derivatives (4)

A suitable aromatic or heterocyclic aldehydes, (Table 1), (0.01 mol), cyclohexanone (0.01 $\mathrm{mol})$ and urea, thiourea or guanidine $\mathrm{HCl}(0.02 \mathrm{~mol})$ in $20 \mathrm{~mL}$ of ethanol in the presence of $(1 \mathrm{~g})$ sodium ethoxide were mixed in a round-bottomed flask. The mixture was refluxed for the time needed to complete the reaction (Monitored by TLC), after cooling to room temperature, the reaction was quenched with $20 \mathrm{~cm}^{3} \mathrm{H}_{2} \mathrm{O}$. The pure product separated, filtered off and recrystallized using ethanol to give the compounds $(\mathbf{4 a - d}, \boldsymbol{i}, \boldsymbol{l}, \boldsymbol{n})$.

\section{Microwave assisted method}

Synthesis of 8-(substituted arylidene) -4- (substituted phenyl) quinazolin-2-(1H)-one derivatives (4)

Suitable aromatic or heterocyclic aldehydes (Table 2), (0.01 mol), cyclohexanone, (0.01 mol) and urea, thiourea or guanidine $\mathrm{HCl}$ were added in Pyrex flask. The mixture was irradiated in an ultrasonic cleaner for the time needed to complete the reaction (Monitored by TCL). After cooling to room temperature, the reaction mixture was quenched with 20 $\mathrm{cm}^{3} \mathrm{H}_{2} \mathrm{O}$. The pure product separated, filtered off and recrystallized using ethanol to give the compounds (4a-r).

\section{Physical and spectral data for the compounds}

8-(2-Chlorobenzylidene)-4-(4-chlorophenyl) -3, 4, 5, 6, 7, 8-hexahydro quinazolin2(1H)-one (4a)

Pale yellow crystal, IR (KBr cm$\left.{ }^{-1}\right) 3025(\mathrm{C}-\mathrm{H}), 1490(\mathrm{C}=\mathrm{C}), 1327$ (C-N), 1689 (N-C=O); 3190(NH); 3045 (Ar-CH), 835(C-Cl); ${ }^{1} \mathrm{H}$ NMR ( $\left.\delta \mathrm{ppm}\right)(400 \mathrm{MHz}$, DMSO-d6): d = 9.0(bs1H, NHCO); 6.64 (1H,C=C); 5.65(1H, CH);1.37-1.96(m 6H, 3×CH2); 7.0-7.24 (m 8H, Ar-H); ${ }^{13} \mathrm{C}$ NMR (100 MHz, DMSO-d6):d = 150.3, 141.3, 140.6,133.1, 129.4, 128.8, 128.7, 128.4,127.8, 127.7 126.8123.9,116.2, 60.1, 30.4, 22.5; MS (ESI): $\mathrm{m} / \mathrm{z}=384.8(17.6)$, 385.8(23), 386.8(64.3).

8-(2-Nitrobenzylidene)-4-(4-nitrophenyl)-3,4,5,6,7,8-hexahydroquinazolin-2 (1H) one (4b)

Yellow crystal, IR (KBr cm$\left.{ }^{-1}\right), 3022(\mathrm{C}-\mathrm{H}), 1490$ (C=C), 1320 (C-N), 1660(N-C=O); 3340(NH); 3020(ArCH); ${ }^{1} \mathrm{H}$ NMR( $(\mathrm{ppm})(400 \mathrm{MHz}, \mathrm{DMSOd} 6): \mathrm{d}=6.03$ (bs1H, NHCO), $5.56(1 \mathrm{H}, \mathrm{CH}) ; 6.93(1 \mathrm{HC}=\mathrm{C}) ; 1.37-1.96\left(\mathrm{~m} 6 \mathrm{H}, 3 \times \mathrm{CH}_{2}\right) ; 7.328 .14(\mathrm{~m} 8 \mathrm{H}, \mathrm{ArH}) ;{ }^{13} \mathrm{C} \mathrm{NMR}$ (100MHz, DMSOd6): $\mathrm{d}=150.3,147.0,146.1,140.6,137.5,134.8,134.7,130.0,128.9$, $127.9,127.7,127.3,121.0,120.9,116.2,51.5,30.4,24.6,22.5 ; \mathrm{MS}(\mathrm{ESI}): \mathrm{m} / \mathrm{z}=406.18$ (11.3), 407.18(23.1), 408.18(3.8). 
8-(4-Methoxybenzylidene)-4-(4methoxyphenyl)-3,4,5,6,7,8-hexahydroquinazolin-2 (1H) one (4c)

Orange crystal, IR (KBr cm$\left.{ }^{-1}\right)$ - $3015(\mathrm{C}-\mathrm{H}), 1490$ (C=C), 1340 (C-N), 1675(N-C=O); 3300(NH); 3037 (Ar-CH), ${ }^{1} \mathrm{H}$ NMR ( $\delta$ ppm) (400 MHz, DMSO-d6):d=4.59(1H,CH); 6.3(1H,C=C),1.37-1.96(m6H,3×CH2); 2.0 $(1 \mathrm{H}, \mathrm{NH}), 3.73(\mathrm{~m} 6 \mathrm{H}, 2 \times \mathrm{CH} 3) ; 6.65-7.17(\mathrm{~m} 8 \mathrm{H}$, Ar-H);); ${ }^{13} \mathrm{C}$ NMR (100 MHz, DMSO-d6):d=159.0, 157.9, 144.3, 132.2, 130.3, 129.2, 129.0, 114.7, 114.1, 84.5, 55.9, 54.3, 41.4, 26.8, 22.7, 21.2.; MS(ESI): $\mathrm{m} / \mathrm{z}$ e $=565.21(10.2)$ 566.21(25), 567.21(2.8).

8-(2-Chlorobenzylidene)-4-(4-chlorophenyl)-3, 4, 5, 6, 7, 8-hexahydroquinazolin-2 (1H) thione $(\mathbf{4 d})$

Yellow crystal, IR (KBr cm$\left.{ }^{-1}\right)-3035(\mathrm{C}-\mathrm{H}), 1475$ (C=C), 1319 (C-N);1272(N-C=S); 3320

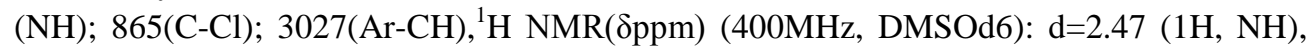
$2.0(1 \mathrm{H}, \mathrm{NH}), 4.59(1 \mathrm{H}, \mathrm{CH}), 6.64(1 \mathrm{H}, \mathrm{C}=\mathrm{C}), 1.37-1.96\left(\mathrm{~m} 6 \mathrm{H}, 3 \times \mathrm{CH}_{2}\right) ; 7.00-7.24(\mathrm{~m} 8 \mathrm{H}, \mathrm{ArH})$; MS(ESI): $m / z=414.07(13.5), 415.07$ (68.6), 416.07 (24.0)

8-(2-Nitrobenzylidene)-4-(4-nitrophenyl)-3,4,5,6,7,8-hexahydroquinazolin-2(1H) thione $\mathbf{( 4 g )}$

Pale yellow crystal, IR (KBr cm $\left.{ }^{-1}\right)-3022(\mathrm{C}-\mathrm{H}) ; 1490$ (C=C); 1327 (C-N); 1235(N-C=S); 3320(NH); 3025(Ar-CH), ${ }^{1} \mathrm{H}$ NMR $(\delta p p m)$ (400MHz, DMSOd6): $\mathrm{d}=2.0(1 \mathrm{H}, \mathrm{NH}) ; 4.59$ $(1 \mathrm{H}, \mathrm{CH}) ; 6.37(1 \mathrm{H}, \mathrm{C}=\mathrm{C}) ; 3.73(3 \mathrm{H}, \mathrm{CH}), 3.73(3 \mathrm{H}, \mathrm{CH}) ; 1.371 .96\left(\mathrm{~m} 6 \mathrm{H}, 3 \times \mathrm{CH}_{2}\right) ; 6.65-$ 7.19(8H, Ar-H); MS (ESI) :m/z=392.16(60)393.16 (26), 394.16 (4.5).

8-(4-Bromobenzylidene)-4-(4-bromophenyl)-3,4,5,6,7,8,-hexahydroquinazolin$2(1 \mathrm{H})$ thione $(4 \mathbf{i})$

Deep yellow crystal, IR (KBr cm $\left.{ }^{-1}\right)-3012(\mathrm{C}-\mathrm{H}) ; 1490(\mathrm{C}=\mathrm{C}) ; 1335(\mathrm{C}-\mathrm{N}) ; 1643(\mathrm{C}=\mathrm{N})$; 3320 (NH);3035 (Ar-CH),1255(NC=S);655(C-Br); ${ }^{13} \mathrm{C}$ NMR(100 MH, DMSOd6):d=174.5,142.2, $140.7,140.6,134.2,131.6,129.2,128.6,123.9,122.3,121.1,65.2,30.4,25.6,22.5) ; M S(E S I): m / z=$ 489.95(20),487.95(35.2); 491.95(2.1).

4-(Thiophen-2-yl)-8-(thiophen-2-ylmethylene)-3,4,5,6,7,8-hexahydroquinazolin$2(1 \mathrm{H})$ thione (4I)

Brown crystal, IR (KBr cm$\left.{ }^{-1}\right) 3020(\mathrm{C}-\mathrm{H}) ; 1488(\mathrm{C}=\mathrm{C}) ; 1338(\mathrm{C}-\mathrm{N}) 3278(\mathrm{NH}) ; 1220(\mathrm{NC}=\mathrm{S})$; ${ }^{13} \mathrm{C}$ NMR (100MHz, DMSOd6):d=174.5, 146.6, 140.7, 138.4, 137.8, 130.5, 128.2, 127.1, 126.9, 126.7, 123.6,120.0, 114.0,60.6,30.4,25.6,22.5.

8-(4-Hydroxybenzylidene)-4-(4-hydroxyphenyl)-1,2,3,4,5,6,7,8-octahydroquina-zolin 2-amine (4q)

yellowish brown crystal, IR (KBr cm$\left.{ }^{-1}\right)-3058(\mathrm{C}-\mathrm{H}) ; 1477$ (C=C); $1315(\mathrm{C}-\mathrm{N})$; 3182(NH); 3367(NH2);3058(Ar-CH),3799(OH); ${ }^{1} \mathrm{H}$ NMR( $(\mathrm{pppm})(400 \mathrm{MHz}, \mathrm{DMSOd} 6): \mathrm{d}=2.0(1 \mathrm{H}, \mathrm{NH})$; 10.1(1H,OH,phenolic);4.59(1H,CH);6.37(1HC=C);1.37-1.96- $(\mathrm{m} 6 \mathrm{H}, 3 \times \mathrm{CH} 2) ; 6.61-7.13$ (m8H,Ar-H).

The spectral data of compounds ( $\mathbf{4 a}-\boldsymbol{d}, \boldsymbol{i}, \boldsymbol{I}$ and $\mathbf{4 n}$ ) which synthesized by method A and B were found to be identical. The IR, ${ }^{1} \mathrm{H}$ NMR, ${ }^{13} \mathrm{C}$ NMR, mass spectroscopy and elemental analyses for the new compound is in accordance with the assigned structures. 


\section{Results and Discussion}

Ultrasound has increasingly been used in organic synthesis in the last three decades. In this work, total eighteen derivatives of 8-(substituted arylidene)-4-(substituted phenyl) quinazoline-2-(1H)-one were prepared by using a Biginelli type three component one-pot cyclocondensation of aromatic or heterocyclic aldehydes (1), cyclohexanone (2) and urea, thiourea or guanidine $\mathrm{HCl}(\mathbf{3})$ in ethanol and sodium ethoxide (4a-d,i,l,n) or under solvent free and microwave assistant (Scheme 1 and 2)

$\mathrm{ArCHO}+$

(1)

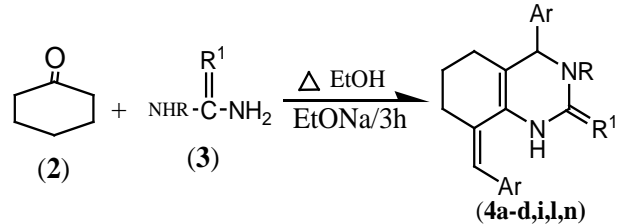

\section{Scheme1}

ArCHO+<smiles>O=C1CCCCC1</smiles>

(2)

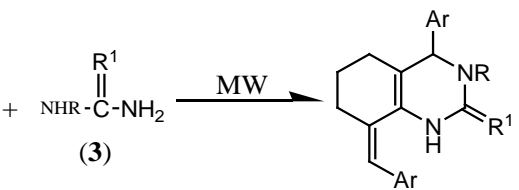

$(4 a-r)$

\begin{tabular}{cccccc}
\hline $\mathrm{Ar}$ & $\mathrm{R}$ & $\mathrm{R}^{1}$ & $\mathrm{Ar}$ & $\mathrm{R}$ & $\mathrm{R}^{1}$ \\
\hline $\mathrm{a}-\mathrm{C}_{6} \mathrm{H}_{4} \mathrm{Cl}-2$ & $\mathrm{H}$ & $\mathrm{O}$ & $\mathrm{H}$ & $\mathrm{S}$ \\
$\mathrm{b}-\mathrm{C}_{6} \mathrm{H}_{4} \mathrm{NO}_{2}-2$ & $\mathrm{H}$ & $\mathrm{O}$ & & & \\
$\mathrm{c}-\mathrm{C}_{6} \mathrm{H}_{4} \mathrm{OCH}_{3}-4$ & $\mathrm{H}$ & $\mathrm{O}$ & & $\mathrm{H}$ & $\mathrm{S}$ \\
$\mathrm{d}-\mathrm{C}_{6} \mathrm{H}_{4} \mathrm{Cl}-2$ & $\mathrm{CH}_{3}$ & $\mathrm{~S}$ & & & \\
$\mathrm{e}-\mathrm{C}_{6} \mathrm{H}_{4} \mathrm{Cl}-2$ & $\mathrm{H}$ & $\mathrm{S}$ & & & \\
$\mathrm{f}-\mathrm{C}_{6} \mathrm{H}_{4} \mathrm{NO}_{2}-2$ & $\mathrm{H}$ & $\mathrm{S}$ & & & \\
g- $\mathrm{C}_{6} \mathrm{H}_{4} \mathrm{NO}_{2}-2$ & $\mathrm{H}$ & $\mathrm{S}$ & $n-\mathrm{C}_{6} \mathrm{H}_{4} \mathrm{Cl}-2$ & $\mathrm{H}$ & $\mathrm{NH}$ \\
$\mathrm{h}-{ }_{6} \mathrm{H}_{4} \mathrm{OCH}_{3}-4$ & $\mathrm{CH}$ & $\mathrm{S}$ & $o-\mathrm{C}_{6} \mathrm{H}_{4} \mathrm{NO}_{2}$ & $\mathrm{H}$ & $\mathrm{NH}$ \\
$\mathrm{i}-\mathrm{C}_{6} \mathrm{H}_{4} \cdot \mathrm{Br}-4$ & $\mathrm{H}$ & $\mathrm{S}$ & $p-\mathrm{C}_{6} \mathrm{H}_{4} \mathrm{OCH}-4$ & $\mathrm{H}$ & $\mathrm{NH}$ \\
j- $\mathrm{C}_{6} \mathrm{H}_{4} \mathrm{NH}-4$ & $\mathrm{H}$ & $\mathrm{S}$ & $q-\mathrm{C}_{6} \mathrm{H}_{4} \mathrm{OH}-4$ & $\mathrm{H}$ & $\mathrm{NH}$ \\
k- $\mathrm{C}_{6} \mathrm{H}_{4} \mathrm{OH}-4$ & $\mathrm{H}$ & $\mathrm{S}$ & $t-\mathrm{C}_{6} \mathrm{H}_{4} \mathrm{Br}-2$ & $\mathrm{H}$ & $\mathrm{NH}$ \\
\hline
\end{tabular}

\section{Scheme 2}

As shown in Table 1, aromatic aldehydes bearing functional groups (for example $\mathrm{OCH}_{3},-\mathrm{Cl},-\mathrm{Br},-\mathrm{NO} 2$ and $-\mathrm{NH}_{2}$ ) react smoothly to give the corresponding products in good yields (80-85\%).

Table 1. Synthesis of Biginelli-type product 4

\begin{tabular}{cccccccc}
\hline Entry & $\mathrm{Ar}$ & Product & $\mathrm{R}^{1}$ & $\mathrm{R}$ & $\begin{array}{c}\text { Molecular } \\
\text { formula }\end{array}$ & $\begin{array}{c}\text { Time } \\
\text { min }\end{array}$ & $\begin{array}{c}\text { Yield } \\
\%\end{array}$ \\
\hline 1 & $\mathrm{C}_{6} \mathrm{H}_{4}-\mathrm{Cl}-2$ & $\mathbf{4 a}$ & $\mathrm{O}$ & $\mathrm{H}$ & $\mathrm{C}_{21} \mathrm{H}_{18} \mathrm{C}_{12} \mathrm{~N}_{2} \mathrm{O}$ & 60 & 85 \\
2 & $\mathrm{C}_{6} \mathrm{H}_{4}-\mathrm{NO}_{2-2}$ & $\mathbf{4 b}$ & $\mathrm{O}$ & $\mathrm{H}$ & $\mathrm{C}_{21} \mathrm{H}_{18} \mathrm{~N}_{4} \mathrm{O}_{5}$ & 30 & 85 \\
3 & $\mathrm{C}_{6} \mathrm{H}_{4}-\mathrm{OCH}_{3}-4$ & $\mathbf{4 c}$ & $\mathrm{O}$ & $\mathrm{H}$ & $\mathrm{C}_{23} \mathrm{H}_{24} \mathrm{~N}_{2} \mathrm{O}_{3}$ & 60 & 85 \\
4 & $\mathrm{C}_{6} \mathrm{H}_{4}-\mathrm{Cl}-2$ & $\mathbf{4 d}$ & $\mathrm{S}$ & $\mathrm{H}$ & $\mathrm{C}_{21} \mathrm{H}_{18} \mathrm{C}_{12} \mathrm{~N}_{2} \mathrm{~S}$ & 60 & 85 \\
5 & $\mathrm{C}_{6} \mathrm{H}_{4}-\mathrm{Br}-4$ & $\mathbf{4 i}$ & $\mathrm{S}$ & $\mathrm{H}$ & $\mathrm{C}_{21} \mathrm{H}_{18} \mathrm{Br}_{2} \mathrm{~N}_{2} \mathrm{~S}$ & 90 & 80 \\
6 & - & $\mathbf{4 l}$ & $\mathrm{S}$ & $\mathrm{H}$ & $\mathrm{C}_{17} \mathrm{H}_{16} \mathrm{~N}_{2} \mathrm{~S}_{3}$ & 90 & 80 \\
7 & $\mathrm{C}_{6} \mathrm{H}_{4}-\mathrm{Cl}-2$ & $\mathbf{4 n}$ & $\mathrm{NH}$ & $\mathrm{H}$ & $\mathrm{C}_{22} \mathrm{H}_{23} \mathrm{C}_{12} \mathrm{~N}_{3}$ & 60 & 80 \\
\hline
\end{tabular}


Moreover, synthesis of 8-(substituted- benzylidene)-4-(substituted-phenyl) 3,4,5,6,7,8hexahydroquinazolin-2(1H)one or thione derivatives and 8-(substituted-benzylidene)-4(substituted-phenyl)1,2,3,4,5,6,7,8-octahydro quinazolin-2-amine derivatives using the one pot microwave- assisted was applied.

It is well noticed that the reaction of benzaldehyde derivatives (1), cyclohexanone (2) and urea, thiourea or guanidine. $\mathrm{HCl}(3)$ was successful and completed within 1-2 min. The overall yields were found in the range of $85-99 \%$ (Table 2). Thus microwave assisted synthesis of compounds (4a-r) were found to be time specific and compare to the classical Biginelli method, and the overall yields were found to be much higher than conventional method route of synthesis. The results are summarized (Table 2). Apparently, under these conditions, the nature of the substituent in the aromatic moiety does not affect significantly the yield of the reactions. As can be seen from data in Table 1,2 in all cases studied, the three-component reaction with both aromatic aldehydes carrying either electron-donating or electron-withdrawing subsistent, hetero aromatic aldehydes, proceeded smoothly giving the corresponding quinazolin-2(1H)one derivatives in high yields.

Table 2. Synthesis of Biginelli-type product 4 under microwave irradiation

\begin{tabular}{|c|c|c|c|c|c|c|c|}
\hline Entry & $\mathrm{Ar}$ & $\mathrm{R}^{1}$ & $\mathrm{R}$ & Product & $\begin{array}{l}\text { Time } \\
\text { (Sec) }\end{array}$ & $\begin{array}{c}\text { Yield } \\
\%\end{array}$ & $\begin{array}{c}\text { Molecular } \\
\text { formula }\end{array}$ \\
\hline 1 & $\mathrm{C}_{6} \mathrm{H}_{4} \mathrm{Cl}-2$ & $\mathrm{O}$ & $\mathrm{H}$ & $4 a$ & 120 & 90 & $\mathrm{C}_{21} \mathrm{H}_{18} \mathrm{C}_{12} \mathrm{~N}_{2} \mathrm{O}$ \\
\hline 2 & $\mathrm{C}_{6} \mathrm{H}_{4}-\mathrm{NO}_{2-2}$ & $\mathrm{O}$ & $\mathrm{H}$ & $4 b$ & 120 & 95 & $\mathrm{C}_{21} \mathrm{H}_{18} \mathrm{~N}_{4} \mathrm{O}_{5}$ \\
\hline 3 & $\mathrm{C}_{6} \mathrm{H}_{4}-\mathrm{OCH}_{3}-4$ & $\mathrm{O}$ & $\mathrm{H}$ & $4 c$ & 60 & 95 & $\mathrm{C}_{23} \mathrm{H}_{24} \mathrm{~N}_{2} \mathrm{O}_{3}$ \\
\hline 4 & $\mathrm{C}_{6} \mathrm{H}_{4} \mathrm{Cl}-2$ & S & $\mathrm{H}$ & 4d & 120 & 98 & $\mathrm{C}_{21} \mathrm{H}_{18} \mathrm{Cl}_{2} \mathrm{~N}_{2} \mathrm{~S}$ \\
\hline 5 & $\mathrm{C}_{6} \mathrm{H}_{4} \mathrm{Cl}-2$ & S & $\mathrm{CH}_{3}$ & $4 e$ & 120 & 92 & $\mathrm{C}_{22} \mathrm{H}_{20} \mathrm{Cl}_{2} \mathrm{~N}_{2} \mathrm{~S}$ \\
\hline 6 & $\mathrm{C}_{6} \mathrm{H}_{4}-\mathrm{NO}_{2-} 2$ & S & $\mathrm{H}$ & $4 f$ & 120 & 98 & $\mathrm{C}_{20} \mathrm{H}_{18} \mathrm{~N}_{4} \mathrm{O}_{4} \mathrm{~S}$ \\
\hline 7 & $\mathrm{C}_{6} \mathrm{H}_{4}-\mathrm{OCH}_{3-4}$ & S & $\mathrm{H}$ & $4 g$ & 60 & 99 & $\mathrm{C}_{23} \mathrm{H}_{24} \mathrm{~N}_{2} \mathrm{O}_{2} \mathrm{~S}$ \\
\hline 8 & $\mathrm{C}_{6} \mathrm{H}_{4}-\mathrm{OCH}_{3}-4$ & S & $\mathrm{CH}_{3}$ & $4 h$ & 60 & 90 & $\mathrm{C}_{22} \mathrm{H}_{21} \mathrm{Br}_{2} \mathrm{~N}_{2} \mathrm{~S}$ \\
\hline 9 & $\mathrm{C}_{6} \mathrm{H}_{4} \mathrm{Br}-4$ & S & $\mathrm{H}$ & $4 i$ & 45 & 95 & $\mathrm{C}_{21} \mathrm{H}_{18} \mathrm{Br}_{2} \mathrm{~N}_{2} \mathrm{~S}$ \\
\hline 10 & $\mathrm{C}_{6} \mathrm{H}_{4-} \mathrm{NH}_{2-} 4$ & S & $\mathrm{H}$ & $4 j$ & 60 & 92 & $\mathrm{C}_{21} \mathrm{H}_{22} \mathrm{~N}_{4} \mathrm{~S}$ \\
\hline 11 & $\mathrm{C}_{6} \mathrm{H}_{4}-\mathrm{OH}-4$ & S & $\mathrm{H}$ & $4 k$ & 60 & 90 & $\mathrm{C}_{21} \mathrm{H}_{20} \mathrm{~N}_{2} \mathrm{O}_{2} \mathrm{~S}$ \\
\hline 12 & & S & $\mathrm{H}$ & 41 & 30 & 85 & $\mathrm{C}_{17} \mathrm{H}_{16} \mathrm{~N}_{2} \mathrm{~S}_{3}$ \\
\hline 13 & & S & $\mathrm{H}$ & $4 m$ & 30 & 85 & $\mathrm{C}_{17} \mathrm{H}_{16} \mathrm{~N}_{2} \mathrm{O}_{2} \mathrm{~S}$ \\
\hline 14 & $\mathrm{C}_{6} \mathrm{H}_{4} \mathrm{Cl}-2$ & $\mathrm{NH}$ & $\mathrm{H}$ & $4 n$ & 120 & 90 & $\mathrm{C}_{22} \mathrm{H}_{23} \mathrm{Cl}_{2} \mathrm{~N}_{3}$ \\
\hline 15 & $\mathrm{C}_{6} \mathrm{H}_{4}-\mathrm{NO}_{2-2} 2$ & $\mathrm{NH}$ & $\mathrm{H}$ & 40 & 120 & 95 & $\mathrm{C}_{21} \mathrm{H}_{21} \mathrm{~N}_{5} \mathrm{O}_{4}$ \\
\hline 16 & $\mathrm{C}_{6} \mathrm{H}_{4}-\mathrm{OCH}_{3-} 4$ & $\mathrm{NH}$ & $\mathrm{H}$ & $4 p$ & 60 & 95 & $\mathrm{C}_{22} \mathrm{H}_{27} \mathrm{~N}_{3} \mathrm{O}_{2}$ \\
\hline 17 & $\mathrm{C}_{6} \mathrm{H}_{4}-\mathrm{OH}-4$ & $\mathrm{NH}$ & $\mathrm{H}$ & $4 q$ & 60 & 85 & $\mathrm{C}_{21} \mathrm{H}_{23} \mathrm{~N}_{3} \mathrm{O}_{2}$ \\
\hline 18 & $\mathrm{C}_{6} \mathrm{H}_{4}-\mathrm{Br}-4$ & $\mathrm{NH}$ & $\mathrm{H}$ & $4 r$ & 60 & 95 & $\mathrm{C}_{21} \mathrm{H}_{21} \mathrm{Br}_{2} \mathrm{~N}_{3}$ \\
\hline
\end{tabular}

\section{Conclusion}

This method is a new and simple modification of the Biginelli-type reaction of aromatic aldehyde, cyclohexanone and urea, thioureaor guanidine to prepare quinazolin-2(1H)one derivatives

\section{References}

1. Shirakawa S and Kobayashi S, Org Lett., 2006, 8, 4939-4942.

2. Ugi I, Domling A and Werner B, J Heterocycl Chem., 2000, 37, 647. 
3. Bagley M C and Lubinu M C, Synthesis, 2006, 8, 1283-1288.

4. Shanthi G and Perumal P T, Tetrahedron Lett., 2009, 50, 3959.

5. Heravi M M, Baghernejad B and Oskooie H A, Tetrahedron Lett., 2009, 50(7), 767-769.

6. $\quad$ Huang X and Zhang T, Tetrahedron Lett., 2009, 50, 208-211.

7. $\quad$ Cui S, Wang J and Wang Y, Org Lett., 2008, 10, 1267.

8. Lu J and Ma H, Synlett., 2000, 63.

9. Reddy C V, Mahesh M, Raju P V K, Babu T R and Reddy V V N, Tetrahedron Lett., 2002, 43, 2657.

10. Ramalinga K, Vijayalakshmi P and Kaimal T N B, Synlett., 2001, 6, 863.

11. Lu J, Bai Y, Wang Z, Yang B and Ma H, Tetrahedron Lett., 2000, 41, 9075-9078.

12. De S K and Gibbs R A, Synthesis, 2005, 1748.

13. Ranu B C, Hazra A and Jana U, J Org Chem., 2000, 65, 6270.

14. Su W, Li J, Zheng Z and Shen Y, Tetrahedron Lett., 2005, 46, 6037.

15. Peng J and Deng Y, Tetrahedron Lett., 2001, 42, 5917-5919.

16. Zhu Y, Huang S and Pan Y, Eur J Org Chem., 2005, 2354.

17. Lei M, Ma L and Hu L, Monatshefte für Chemie, 2010, 141(9), 1005-1008. 